\title{
Finite Element Analysis of Two Dimensional Functionally Graded Thick Rotating Disks
}

\author{
S. Dewangan ${ }^{1}$, L. Sondhi ${ }^{2}$, Jeetendra Kumar Tiwari ${ }^{3}$, \\ Department of Mechanical Engineering, Shri Shankaracharya Technical Campus, SSGI, Bhilai, India
}

\begin{abstract}
The present study deals with the stress and deformation analysis of thick rotating disks made up of two dimensional functionally graded materials (FGMs) by finite element method. Material properties are distributed according to power law distribution and the disks are subjected to clamped-free boundary condition. Material modeling and finite element modeling for the disk is presented and the resulting deformation and stresses are evaluated. The effect of grading index on the deformation and stresses is investigated and presented. The results obtained show that there is a significant reduction of stresses in two dimensional FGM disks as compared to homogeneous and one dimensional FGM disks.
\end{abstract}

Keywords: Two dimensional functionally graded material, Finite element analysis, Thick rotating disk.

\section{INTRODUCTION}

Many engineering components such as gas turbines, gears, turbo-machinery, flywheel systems, centrifugal compressors are modeled as rotating circular disks in the field of marine, mechanical and aerospace industries. The total stresses due to centrifugal load plays a very important role on their strength and safety. Thus, optimization of total stresses and displacement fields is an important task. Functionally gradation of the material properties optimize the component strength by controlling the changes of the local material properties. Depending on the function of the component, it is possible to utilize one, two or three-directional distributions of the material properties.

A few researchers have reported work on analysis of two dimensional FGM disks by analytical and finite element method. Zafarmand et al. [1] worked on elastic analysis of twodimensional functionally graded rotating annular and solid disks with variable thickness. Axisymmetric conditions are assumed for the twodimensional functionally graded disk and the graded finite element method (GFEM) has been applied to solve the equations. Shariyat et al. [2] worked on Three-dimensional bending and stress analyses of the rotating two-directional functionally graded annular/circular plates or disks. Compatible Hermitian elements are employed to develop a consistent formulation and avoid jumps in the stress components at the elements interfaces. Eraslan et al. [3] has obtained analytical solutions for the elastic plastic stress distribution in rotating variable thickness annular disks. Thickness of the disks have parabolic variation and the analysis is based on the tresca's yield criterion. Bayat et al. [4] reported work on analysis of a variable thickness FGM rotating disk. Material properties vary according to power law and the disk is subjected to both the mechanical and thermal loads. Afsar et al. [5] have analyzed a rotating FGM circular disk subjected to mechanical as well as thermal load by finite element method. The disk has exponentially varying material properties in radial direction. The disk is subjected to a thermal load along with centrifugal load due to non uniform temperature distribution. An analytical thermoelasticity solution for a disc made of functionally graded materials (FGMs) is presented by Callioglu [6]. Bayat et al. [7] investigated displacement and stress behavior of a functionally graded rotating disk of variable thickness by semi analytical method. Radially varying one dimensional FGM is taken and material properties vary according to power law and Mori-Tanaka scheme. Disk subjected to centrifugal load is analyzed for fixed boundary condition at inner surface and free boundary condition at outer surface. Callioglu et al. [8] have analyzed thin FGM disks. Density and Modulus of elasticity varies according to power law in an FGM of aluminum ceramic and the effect of grading parameter on displacement and stresses are investigated. Callioglu et al. [9] has analyzed functionally graded rotating annular disk subjected to internal pressure and various temperature distributions such as uniform temperature, linearly increasing and decreasing temperature in radial direction. Sharma et al. [10] worked on the analysis of stresses, displacements and strains in a thin circular functionally graded material (FGM) disk by finite element method. The disk is subjected to mechanical as well as thermal loads. Ali et al. [11] reported study on the elastic analysis of two 
sigmoid FGM rotating disks. Metal-Ceramic-Metal disk is analyzed for both uniform and variable thickness disks and effect of grading index on the displacement and stresses are investigated. Nejad et al. [12] have found closed-form Analytical solution of an exponentially varying FGM disk which is subjected to internal and external pressure.

In a recent work Rosyid et al. [13] worked on finite element analysis of nonhomogeneous rotating disc with arbitrarily variable thickness. Three types of grading law namely Power law, sigmoid and exponential distribution law is considered for the volume fraction distributions. Zafarmand et al. [14] investigated nonlinear elasticity solution of functionally graded nanocomposite rotating thick disks with variable thickness reinforced with single-walled carbon nanotubes (SWCNTs). The governing nonlinear equations derived are based on the axisymmetric theory of elasticity with the geometric nonlinearity in axisymmetric complete form and are solved by nonlinear graded finite element method (NGFEM).

In present research work rotating thick disks of two dimensional FGM, having clampedfree boundary condition are analyzed. Material properties of the disks are graded along the radial as well axial direction according to power law distribution by element based material grading. A finite element formulation based on principle of stationary total potential is presented for the rotating disks and the effect of grading parameters $n_{r}$ and $n_{z}$ on the deformation and stresses is investigated.

\section{MATERIAL MODELING}

Material properties are graded along the radial direction and axial direction using two dimensional power law. At inner radius there is FGM of two metals and at outer radius there is FGM of two ceramic, whose properties are given in Table 1 [1]. Volume frac01tion of the metal 1, metal 2, ceramic 1 and ceramic 2 are given as:

$$
\begin{aligned}
& V_{m 1}(r, z)=\left\lfloor 1-\left(\frac{r-a}{b-a}\right)^{n_{r}}\right\rceil\left\lceil 1-\left(\frac{z+h}{2 h}\right)^{n_{z}}\right\rceil \\
& V_{m 2}(r, z)=\left\lfloor 1-\left(\frac{r-a}{b-a}\right)^{n_{r}}\right\rceil\left(\frac{z+h}{2 h}\right)^{n_{z}} \\
& V_{c 1}(r, z)=\left(\frac{r-a}{b-a}\right)^{n_{r}}\left\lfloor 1-\left(\frac{z+h}{2 h}\right)^{n_{z}}\right\rfloor \\
& V_{c 2}(r, z)=\left(\frac{r-a}{b-a}\right)^{n_{r}}\left(\frac{z+h}{2 h}\right)^{n_{z}}
\end{aligned}
$$

Here $V_{m 1}, V_{m 2}, V_{c 1}$ and $V_{c 2}$ are volume fraction of the metal 1 , metal 2 , ceramic 1 and ceramic 2 respectively. $a$ and $b$ are inner and outer radius, $h$ is the thickness and $n_{r}$ and $n_{z}$ are non negative volume fraction exponent for distribution in radial as well as in axial direction.

The material property $\varphi$ at any point can be obtained using linear rule of mixture, i.e.

$\varphi=\varphi_{c 1} V_{c 1}+\varphi_{c 2} V_{c 2}+\varphi_{m 1} V_{m 1}+\varphi_{m 2} V_{m 2}$

Where $\varphi_{c l}, \varphi_{c 2}, \varphi_{m l}$ and $\varphi_{m 2}$ are material properties of first ceramic, second ceramic, first metal and second metal respectively.

\section{FINITE ELEMENT FORMULATION}

Rotating disk is an axisymmetric body whose behavior is independent of coordinate $\theta$ of a cylindrical frame of reference $(r, \theta, z)$. Using quadratic quadrilateral element, the displacement vector $\{\varphi\}$ can be obtained as

Table 1.

Material properties of constituent metals and ceramics of FGM disks [Zafarmand et al., 2014].

\begin{tabular}{lll}
\hline Material & $\mathrm{E}(\mathrm{GPa})$ & $\begin{array}{l}\rho \\
\left(\mathrm{kg} / \mathrm{m}^{3}\right)\end{array}$ \\
\hline$M_{1}$ & 115 & 4515 \\
$M_{2}$ & 69 & 2715 \\
$C_{1}$ & 440 & 3210 \\
$C_{2}$ & 380 & 3470
\end{tabular}

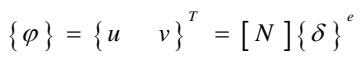


where $u$ and $v$ are the components of displacement in radial and axial direction respectively, $[N]$ is the matrix of linear shape functions and $\{\delta\}$ is the nodal displacement vector, given as:

$[N]=\left[\begin{array}{cccccccc}N_{1} & 0 & N_{2} & 0 & N_{3} & 0 & N_{4} & 0 \\ 0 & N_{1} & 0 & N_{2} & 0 & N_{3} & 0 & N_{4}\end{array}\right]$
$\{\delta\}^{e}=\left\{\begin{array}{llllllll}u_{1} & v_{1} & u_{2} & v_{2} & u_{3} & v_{3} & u_{4} & v_{4}\end{array}\right\}^{T}$

Shape functions in natural coordinates are given as [15]:

$$
\begin{aligned}
& N_{1}=\left(\frac{1}{4}\right)(1-\xi)(1-\eta) \\
& N_{2}=\left(\frac{1}{4}\right)(1+\xi)(1-\eta) \\
& N_{3}=\left(\frac{1}{4}\right)(1+\xi)(1+\eta) \\
& N_{4}=\left(\frac{1}{4}\right)(1-\xi)(1+\eta)
\end{aligned}
$$

In generalized matrix notation, stress-strain relation can be written as:

$\{\sigma\}=[D(r, z)]\{\varepsilon\}$

where $D(r, \mathrm{z})$ is stress-strain relationship matrix and is a function of radius $r$ and thickness parameter $z$.

$$
\begin{aligned}
& \{\sigma\}=\left\{\begin{array}{llll}
\sigma_{r} & \sigma_{\theta} & \sigma_{z} & \tau_{r}
\end{array}\right\}^{T} \\
& \{\varepsilon\}=\left\{\begin{array}{lllll}
\varepsilon_{r} & \varepsilon_{\theta} & \varepsilon_{z} & \gamma_{r z}
\end{array}\right\}^{T}
\end{aligned}
$$

$$
D(r, z)=\frac{(1-v) E(r, z)}{(1+v)(1-2 v)}\left[\begin{array}{cccc}
1 & \frac{v}{(1-v)} & \frac{v}{(1-v)} & 0 \\
\frac{v}{(1-v)} & 1 & \frac{v}{(1-v)} & 0 \\
\frac{v}{(1-v)} & \frac{v}{(1-v)} & 1 & 0 \\
0 & 0 & 0 & \frac{1-2 v}{2(1-v)}
\end{array}\right]
$$

When the disk rotates, it experiences a distributed force over its volume. Under these force when disk is properly supported, it undergoes deformation and stores internal strain energy $U$, which is given by [15]:

$U=\frac{1}{2} \int_{v}\{\varepsilon\}^{r}\{\sigma\} d v$

The potential of external body and surface force is given by

$W=-\int_{v}\{\delta\}^{T}\left\{q_{v}\right\} d v-\int_{s}\{\delta\}^{T}\left\{q_{s}\right\} d s$

Using the Principle of stationary total potential (PSTP) the total potential is set to be stationary with respect to small variation in the nodal degree of freedom that is:

$\frac{\partial \pi_{p}}{\partial\{\delta\}^{T}}=0$

which gives system level equation for disk as:

$[K]\{\delta\}=\{F\}$

where

$$
\begin{aligned}
& {[K]=\sum_{n=1}^{N}[K]^{e}=\text { Globel Stiffness matrix }} \\
& \{F\}=\sum_{n=1}^{N}[f]^{e}=\text { Globel load vector }
\end{aligned}
$$




\section{RESULTS AND DISCUSSION}

\subsection{Validation}

A numerical problem of uniform thickness rotating disk of reference [1] is reconsidered for the purpose of validation of the present work. The disk has two dimensional power law distribution of material properties according to Eq. (1-5). Fig 1 shows the comparison of non dimensional circumferential stress in radial direction for $z=0$, $n_{r}=3$ and $n_{z}=2$.

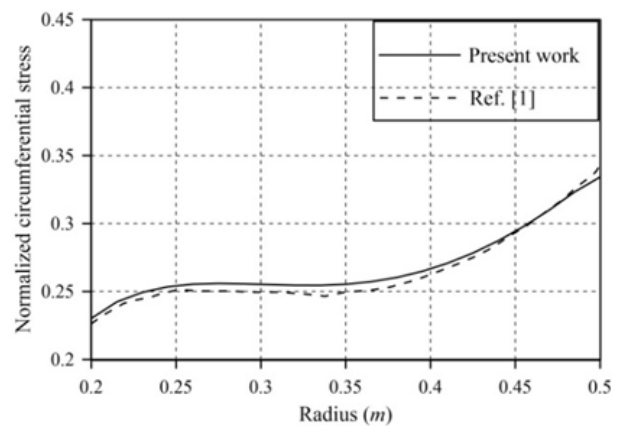

Figure 1 Comparison of circumferential stress distribution along radial direction with ref [1]

\subsection{Numerical results and discussion}

In this section rotating annular disks of uniform thickness profiles having clamped-free boundary condition are analyzed. Disks are made of two dimensional FGM of two metal and two ceramics whose young's modulus and densities vary along radial and axial direction according to Eq. (1-5). Material properties of individual metal and ceramic are given in Table 1. Fig 3 to Fig 15 shows the distribution of radial displacement, radial stress and circumferential stress respectively for different values of grading parameter $n_{r}$ and $n_{z}$ along radial and axial direction.

Grading index $n_{r}=n_{z}=0$ indicates that the disk is made of homogeneous material while $n_{r}=0$, $n_{z}=1$ and $n_{r}=1, n_{z}=0$ indicates that the disk is made of one dimensional FGM. Values of $n_{r}$ and $n_{z}$ other then this indicates two dimensional FGM.

Figure 2 to Figure 9 shows the effects of grading parameter $n_{z}$ on stresses, strains and deformations for $n_{r}=1$ and 0 . From Figure 2 it can be seen that FGM disk having $n_{r}=1$ and $n_{z}=0$ has maximum radial displacement while the FGM shell having $n_{r}=0$ and $n_{z}=0$ has minimum radial displacement. $n_{r}=0$ and $n_{z}=0$ indicates homogeneous disk made of ceramic 2 and $n_{r}=1$ and $n_{z}=0$ indicates one dimensional FGM whose material properties vary along radial direction and are independent of axial direction. Radial displacement is minimum that is zero at the inner surface which shows the clamped boundary condition applied at the inner radius. From Figure 3 it is observed that radial stress is zero at the outer surface and maximum at the inner surface, which confirms the free boundary condition at outer radius. Homogeneous disk has maximum radial stress while disk having $n_{r}=1$ and $n_{z}=0$ has minimum radial stress. Radial stress is increasing with increasing value of $n_{z}$ except for homogeneous disk. Circumferential stress is approximate same at outer radius for all values of $n_{z}$, except homogeneous disk but differ at the inner radius. Circumferential stress increases with increasing $n_{z}$ apart from homogeneous disk. Disk having $n_{r}=1$ and $n_{z}=3$ has the maximum circumferential stress while disk having $n_{r}=0$ and $n_{z}=0$ has minimum circumferential stress.Von mises stresses have same distribution pattern as circumferential stresses. It is maximum at the inner radius for the disk having $n_{r}=0$ and $n_{z}=0$ while minimum for the disk having $n_{r}=1$ and $n_{z}=0$.

From Figure 6 it can be seen that FGM disk having $n_{r}=1$ and $n_{z}=0$ has maximum radial displacement while the FGM disk having $n_{r}=0$ and $n_{z}=0$ has minimum radial displacement. Radial displacement distribution is approximately linear over the thickness except near the top and bottom surface. Radial displacement is decreasing with increasing value of $n_{z}$ along the axial direction except homogeneous disk. From Figure 7 it is observed that homogeneous disk has maximum radial stress while disk having $n_{r}=1$ and $n_{z}=0$ has minimum radial stress. Radial stress is increasing with increasing value of $n_{z}$. It is also linear over the central thickness zone and increases and decreases gradually near the bottom and top zone respectively. Circumferential stress increases gradually from bottom surface to middle of the thickness while again decreases from middle to top surface gradually. Disk having $n_{r}=0$ and $n_{z}=0$ has the minimum circumferential stress while disk having $n_{r}=1$ and $n_{z}=3$ has maximum circumferential stress.Von mises stresses is maximum near the inner radius and minimum at the outer radius. It is maximum for the disk having $n_{r}=1$ and $n_{z}=3$ and minimum for the disk having $n_{r}=0$ and $n_{z}=0$. It can be observed that von mises stress is increasing with increasing $n_{z}$. 


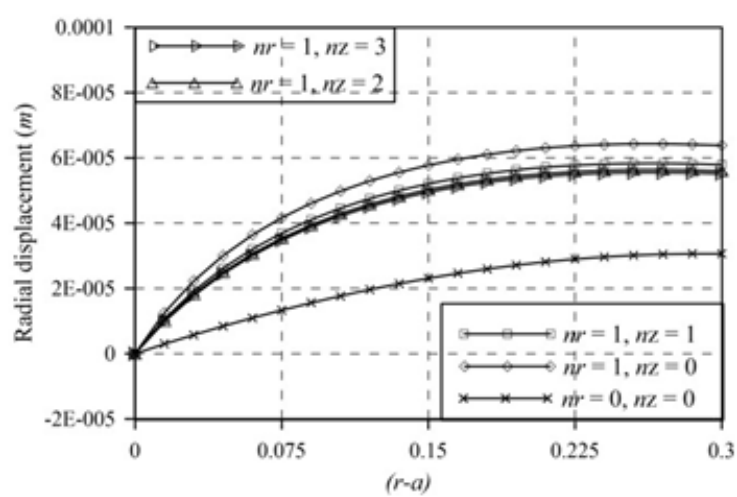

Figure 2 Distribution of radial displacements along ra dial direction at $z=0$ for different values of $n_{z}$

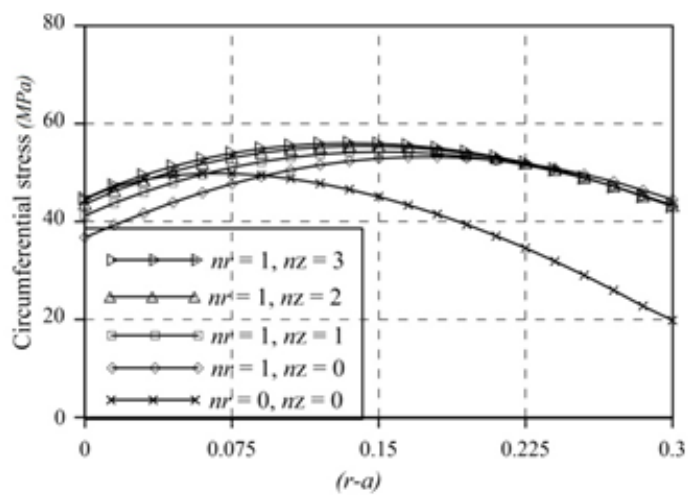

Figure 4 Distribution of circumferential stress along radial direction at $z=0$ for different values of $n$,

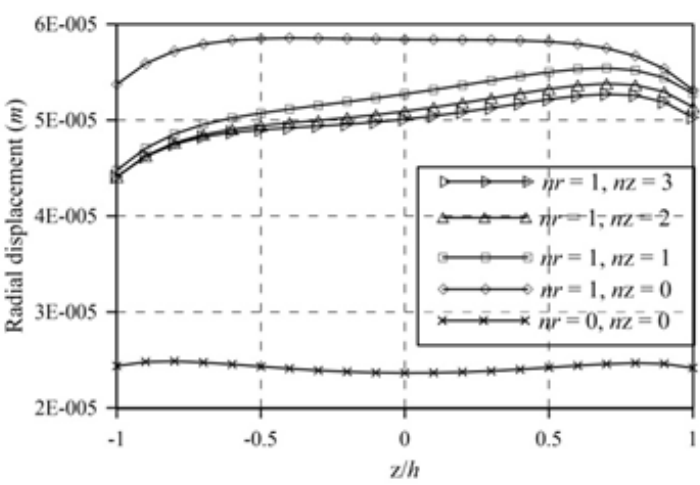

Figure 6 Distribution of radial displacements along axial direction at $r=0.35$ for different values of $n_{z}$

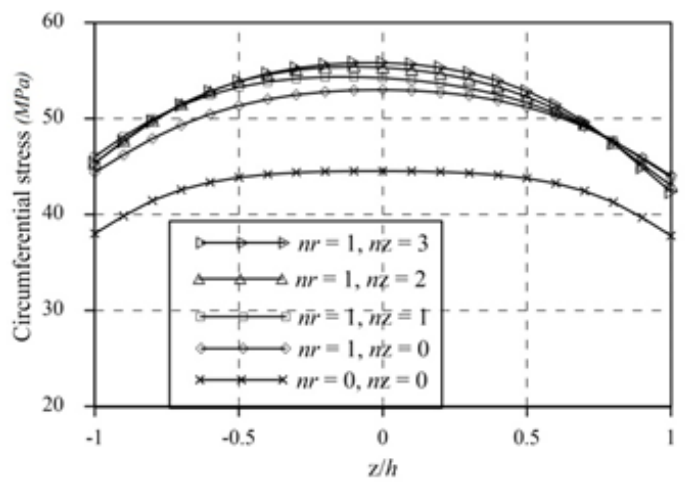

Figure 8 Distribution of circumferential stress along axial direction at $r=0.35$ for different values of $n_{s}$

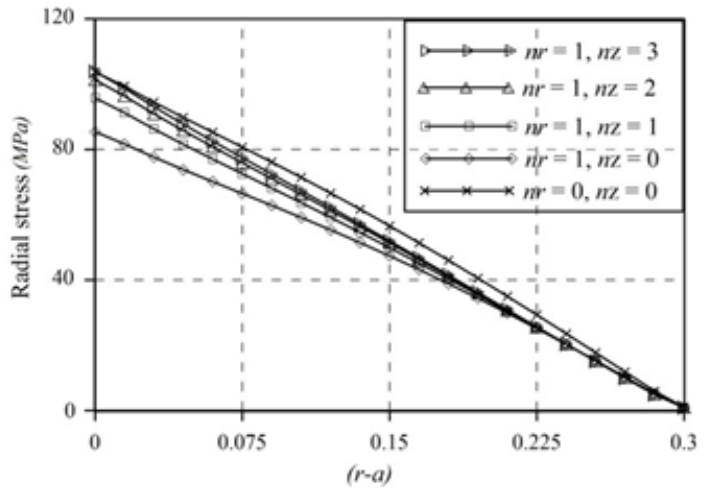

Figure 3 Distribution of radial stress along radial directio: at $z=0$ for different values of $n$.

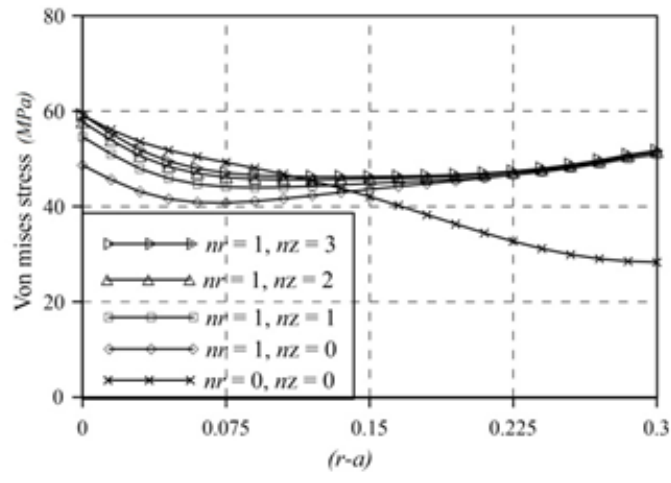

Figure 5 Distribution of von Mises stress along radial direction at $z=0$ for different values of $n_{z}$

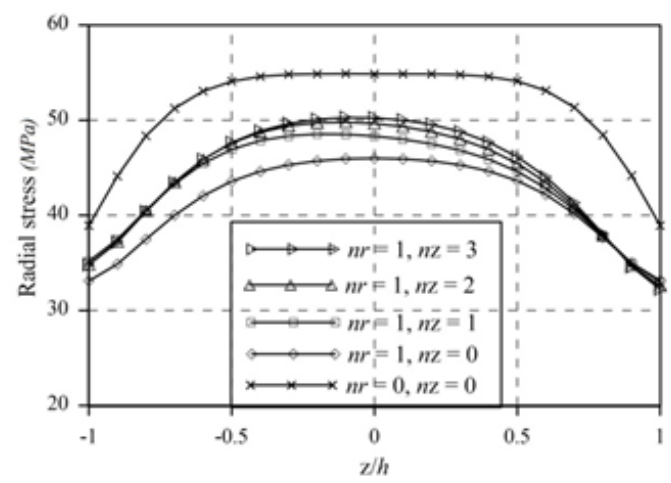

Figure 7 Distribution of ra dial stress along axial direction at $r$ $=0.35$ for different values of $n$

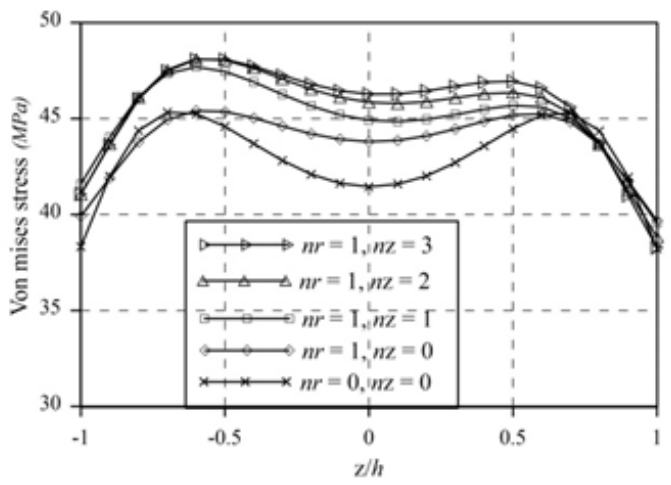

Figure 9 Distribution of von Mises stress along axial direction at $r=0.35$ for different values of $n_{z}$ 


\section{CONCLUSION}

In present work stress and deformation analysis of two dimensional FGM rotating thick disks are presented. Material properties are modeled by power law distribution and are subjected to clamped-free boundary condition. It is observed that there is a significant reduction in stresses and deformation behavior of the two dimensional FGM disks compared to homogeneous disks and one dimensional FGM disks. It is observed that radial stress is maximum as compared circumferential stress. Therefore it is suggested to use radial stress as stress criteria for designing rotating disk of two dimensional FGM. Further it is observed that the disk having $n_{r}=1$ and $n_{z}=0$ has the least radial stress.

\section{REFERENCES}

[1] Zafarmand H, Hassani B (2014) Analysis of two-dimensional functionally graded rotating thick disks with variable thickness. Acta Mech 225: 453-464.

[2] Shariyat M, Mohammadjani R (2013) Threedimensional compatible finite element stress analysis of spinning two-directional FGM annular plates and disks with load and elastic foundation nonuniformities. LAJSS 10: $859-890$.

[3] Eraslan AN (2003) Elastic-plastic deformations of rotating variable thickness annular disks with free, pressurized and radially constrained boundary conditions. IJMS 45: 643-667.

[4] Bayat M, Saleem M, Sahari BB, Hamouda AMS, Mahdi E (2009) Mechanical and thermal stresses in a functionally graded rotating disk with variable thickness due to radially symmetry loads. International Journal of Pressure Vessels and Piping 86: 357-372.

[5] Afsar AM, Go J (2010) Finite element analysis of thermoelastic field in a rotating FGM circular disk. Applied Mathematical Modelling 34: 3309-3320.

[6] Callioglu H (2011) Stress analysis in a functionally graded disc under mechanical loads and a steady state temperature distribution. Sadhana 36: 53-64.

[7] Bayat M, Sahari BB, Saleem M, Dezvareh E, Mohazzab AH (2011) Analysis of Functionally Graded Rotating Disks with Parabolic Concave Thickness Applying an Exponential Function and the Mori-Tanaka Scheme. IOP Conf. Series: Materials Science and Engineering 17: 1-11.

[8] Callioglu H, Sayer M, Demir E (2011) Stress analysis of functionally graded discs under mechanical and thermal loads. Indian
Journal of Engineering \& Material Sciences 18: 111-118.

[9] Callioglu H, Bektas NB, Sayer M (2011) Stress analysis of functionally graded rotating discs: analytical and numerical solutions. Acta Mech. Sin 27: 950-955.

[10] Sharma JN, Sharma D, Kumar S (2012) Stress and strain analysis of rotating FGM Thermoelastic circular disk by using FEM. International Journal of Pure and Applied Mathematics 74: 339-352.

[11] Ali A, Bayat M, Sahari BB, Saleem M, Zaroog OS (2012) The effect of ceramic in combinations of two sigmoid functionally graded rotating disks with variable thickness. Scientific Research and Essays 7: 2174-2188.

[12] Nejad MZ, Abedi M, Lotfian MH, Ghannad M (2013) Elastic analysis of exponential FGM disks subjected to internal and external pressure. Central European Journal of Engineering 3: 459-465.

[13] Rosyid A, Saheb ME, Yahia FB (2014) Stress Analysis of Nonhomogeneous Rotating Disc with Arbitrarily Variable Thickness Using Finite Element Method. Research Journal of Applied Sciences, Engineering and Technology 7: 3114-3125.

[14] Zafarmand H, Kadkhodayan M (2015) Nonlinear analysis of functionally graded nanocomposite rotating thick disks with variable thickness reinforced with carbon nanotubes. Aerospace Science and Technology 41: 47-54.

[15] Seshu P (2003) Text book of finite element analysis, PHI Learning Pvt. Ltd. 